\title{
CASE REPORT An Unusual Case of Foreign Body Aspiration: A Case Report
}

\author{
*K Roy ${ }^{1}$, S K Amin ${ }^{2}$, M Setu ${ }^{3}$, T Khondaker ${ }^{4}$, NS Chowdhury ${ }^{5}$ \\ ${ }^{1}$ Dr. Kuntal Roy, Registrar, Department of Pediatrics, Anwer Khan Modern Medical College \\ ${ }^{2}$ Prof. Syed Khairul Amin, Professor and Head of the Department, Department of Pediatrics Anwer Khan Modern Medical College \\ ${ }^{3}$ Dr. Mumtahina Setu, Assistant Professor, Department of Pediatrics, Anwer Khan Modern Medical College \\ ${ }^{4}$ Dr. Tarannum Khondaker, Registrar, Department of Pediatrics, Anwer Khan Modern Medical College \\ ${ }^{5}$ Dr. Nandita Sur Chowdhury, Lecturer, Department of Microbiology, Anwer Khan Modern Medical College
}

*Corresponding Author

Date of submission: 12 February 2015 Date of acceptance: 26 April 2015

\section{ABSTRACT}

Foreign body aspiration most commonly affects young children, with respiratory symptoms such as wheeze and cough after a choking episode. A careful history and clinical examination can identify those children that need additional investigation including bronchoscopy. However foreign body aspiration can mimic other conditions. The link between choking and subsequent symptoms may not be made by parents. We present a case with a delay in diagnosis, and discuss the appropriate management of suspected foreign body aspiration.

Key Words: Foreign body aspiration, X-ray chest, Bronchoscopy

\section{Introduction}

Foreign bodies in the upper aero-digestive tract are an important cause of morbidity and mortality in the two extremes of life. In the USA in 1985, accidental ingestion of foreign objects led to 170 deaths in infants under 12 months of age (Centers for Disease Control, 1985). Tracheobronchial foreign body aspirations (FBAs) are a frequent cause of respiratory problems in children and results in acute respiratory distress, chronic lung disease and even death. ${ }^{1}$ FBA is a life-threatening event associated with a prohibitive morbidity in children below 3 years of age. It is also the commonest cause of accidental death in infancy. ${ }^{2}$ As opposed to the ingested foreign bodies which gain entrance into the digestive tract; inhaled foreign bodies find their way to get lodged into the trachea-bronchial tree. Although foreign body inhalation into the air passages cannot be claimed a domain of a particular age, but nearly $80 \%$ of these accidents are encountered in the pediatric age group. ${ }^{3}$ Most frequently, aspirated objects are food, which is involved in $75 \%$ of the cases; other organic materials, such as bones, teeth, and plants 7\%, nonorganic materials such as metals and plastics $13 \%$, rocks $1 \%$, and toys or parts of toys $1 \% .4$

Foreign body can settle in hypopharynx $(5 \%)$, larynx $(2-9 \%)$, trachea $(12 \%)$ or bronchus $(83 \%)$. The airway obstruction may be partial or complete. Partial obstruction occurs when the upper airways are partially occluded or obstruction is distal to carina. Patient may present within weeks to months after foreign body aspiration. Most foreign body lodge in periphery, distal to Larynx or Trachea. However foreign body having a sharp or irregular body gets lodged in larynx or Trachea. 5,6

\section{Case Presentation}

A 1 year old immunized Hindu child, 1st issue of a non-consanguineous parents, hailing from Singuir, Manikgonj admitted into pediatric ward of AKMMCH with the complaints of cough \& respiratory distress for last 20 days. According to the statement of the patient's mother, the baby was reasonably well about 20 days back. Then she developed cough which was associated with respiratory distress but not associated with fever or runny nose. For these complaints, she visited to local doctor and pediatrician and diagnosed as a case of bronchopneumonia and treated with several antibiotics. But her cough and respiratory distress didn't relieve at all. On examination, her respiratory rate was $44 / \mathrm{min}, \mathrm{H} / \mathrm{R}$ 120/min, Temp-Normal. On auscultation, breath sound was vesicular and rhonchi was heard over left lung. Other systemic examination reveals no abnormality.

In X-ray chest (AP view in expiration) we found, hyperinflation and hyper- translucency in left lung field (obstructive emphysema), may be due to obstruction in left bronchus. From this x-ray finding we suspected partial obstruction of left lung may be 
due to foreign body inhalation and bronchoscopy was advised. So a rigid bronchoscopy was done under general anesthesia and a foreign body (red long plastic piece which is a part of a toy) was found occluding part of trachea and left principal bronchus. Then the foreign body was removed with forceps. After removal of the foreign body, the baby's condition improved and we released her when she got fully recovered.

\section{Discussion}

Foreign body aspiration is a very frequently occurring situation in pediatric practice; however, it is not always immediately diagnosed, partly because this diagnosis is not frequently in mind, but also because FBA does not have a specific clinical manifestation. ${ }^{7}$ Among the pediatric patients, children between the ages of 12-48 months have been found to be the major victims owing to their poor chewing ability because of the lack of posterior dentition, a tendency to put things into mouth, and a tendency to have frequent vigorous, uninhibited inspirations when startled, laughing or coughing. ${ }^{8}$ Foreign bodies have a tendency to lodge in the right main stem bronchus as it is more vertical and larger in diameter than the left main stem bronchus. However, as in this case report, foreign bodies may enter the left main stem bronchus and, in fact, have been reported in all airway locations. ${ }^{5,8}$

FBA can be result in a great variety of symptoms of varying severity, or it even can be completely asymptomatic. The severity of the complications of FBA is frequently underestimated. Although some studies report a low percentage of serious complications, they are in fact more common than it is normally reported and are more severe in those patients who have the foreign body located in the tracheobronchial tree for a prolonged time. ${ }^{9,10}$

In 1897 Gustav Killer removed a foreign body from lower respiratory tract with a rigid bronchoscope. During 1 st past of $20^{\text {th }}$ century Chevalier Jackson perfected endoscopic technique. Even bronchoscopy, considered a safe technique, carries a risk of complications that has received little emphasis in other reviews. In fact, these potential complications are significant and may occur even in experienced hands, primarily when the foreign body is located in distal areas. ${ }^{11}$ Rigid bronchoscopy is the procedure of choice for retrieval of airway foreign bodies in children. Complications like bronchospasm, foreign body dislodgement, pneumothorax, pneumomediastinum, hydropneumothorax, pneumonia, atelectasis, bronchial stenosis and abscess occur in $22-33 \%$ of patients while tracheal/vocal cord lacerations, subglottic edema and need for surgical retrieval of the FB are seen in 6-8\%. ${ }^{11}$ Since foreign bodies are typically stuck distally in the lower lobe bronchi or the bronchus intermedius, acute presentation is rare; however, life-threatening asphyxia and sudden decompensation secondary to complete obstruction may occur. ${ }^{12}$ Our patient presented with cough which is the most common presenting symptom. Other symptoms include fever, dyspnea. Physical examination of adults with foreign body aspiration is often unrevealing. Stridor, wheezing, or diminished breath sounds may be encountered if the degree of obstruction is severe enough. ${ }^{14}$

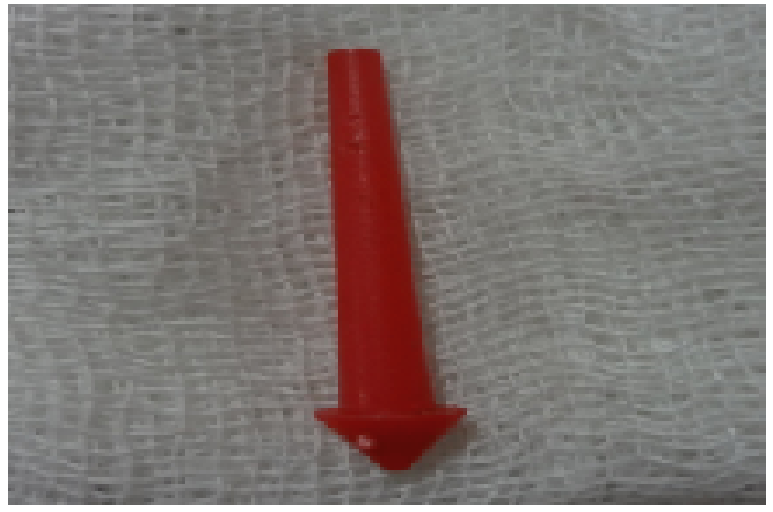

Fig 1: Foreign body (red long plastic piece which is a part of a toy)

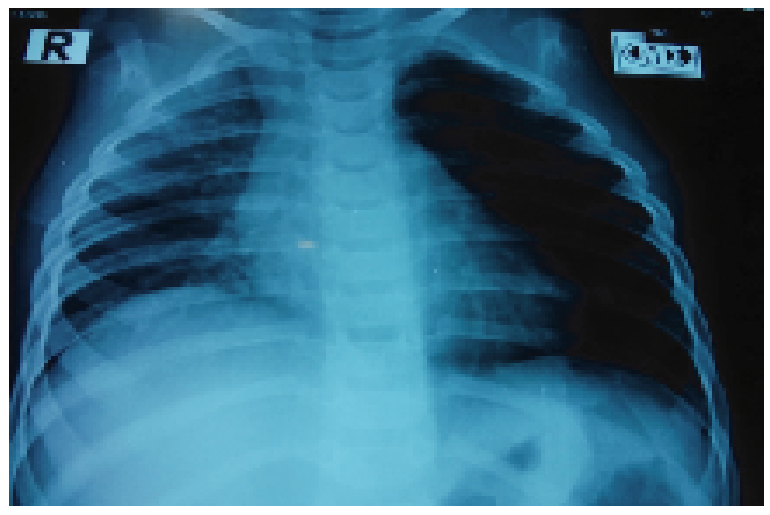

Fig 2: X-ray chest (AP view in expiration): hyperinflation and hyper- translucency in left lung field (obstructive emphysema).

Many foreign bodies are incidentally seen on radiographic imaging ordered for symptoms mistakenly attributed to other medical conditions including asthma and unresolving recurrent pneumonia. ${ }^{12,14}$ If a diagnosis of foreign body aspiration is delayed, a retained foreign body may 
result in unresolving pneumonia, lung abscess, and bronchiectasis. Also, formation of granulation tissue around the foreign body may occur and may resemble bronchogenic carcinoma. ${ }^{14}$

\section{Conclusion}

Unusual foreign body like the case which we reported may miss the diagnosis as there was no history of Foreign Body Inhalation. Moreover, the foreign body was radiolucent. Complete detailed history, clinical and radiological findings helps to detect such case. It is important to diagnose the case as early as possible, so that there may not be any catastrophe.

\section{Conflict of Interest}

The authors have no conflict of interest to anybody.

\section{References}

1. G.M. Zaytoun, P.W. Rouadi, D.H. Baki, Endoscopic management of foreign bodies in tracheobronchial tree: predictive factors for complications. Otolaryngol Head Neck Surg. 2000; 123(3):311-6.

2. Brown BS, Ma H, Dunber JS et al. - Foreign bodies in tracheobroncheal tree in childhood. J Can Assoc Radiol 1963; 14: 158-71.

3. Svensson G. Foreign bodies in tracheobroncheal tree, Special reference to experience in 97 children. Int J Pediatr Otolaryngol 1985; 8:234-51.

4. Causey AL, Talton DS, Miller RC et al. - Aspirated safety pin requiring thoracotomy: report of a case and review. Pediatr Emerg Care 1997; 13:397-400.

5. Diop EM, Tall A, Diout R. Laryngeal foreign body: Management in children in Senegal: Arch pediatric. 2000;7:10-5.
6. Rothman BF, Beockman CR. Foreign bodies in the larynx and tracheobronchial tree in children: A reviewof 225 cases. Ann Otol Rhinol and Laryngol 1980; 89:434-6

7. Cataneo AJ, Reibscheid SM, Ruiz junior RL et al. - Foreign body in the tracheobronchial tree. Clin Pediatr (Phila) 1997;36:701-6.

8. R.E. Black, K.J. Choi, Syme WC et al. - Bronchoscopic removal of aspirated foreign bodies in children. The American Journal of Surgery. 1984; 148(6) 778-781.

9. Black RE, Choi KJ, Syme WC et al. - Bronchoscopic removal of aspirated foreign bodies in children. Am J Surg 1984;31:937-941.

10. Metrangolo S, Monetti C, Meneghini L et al. - Eight years' experience with foreign body aspiration in children: what is really important for a timely diagnosis? J Pediatr Surg 1999;34:1229-31.

11. Zerella JT, Dimler M, Mcgill LC et al. - Foreign body aspiration in children: value of radiography and complications of bronchoscopy. J Pediatr Surg 1998;33:1651-4.

12. A. Aissaoui, N. H. Salem, and A. Chadly, "Unusual foreign body aspiration as a cause of asphyxia in adults: an autopsy case report," The American Journal of Forensic Medicine and Pathology. 2012; 33(3): 284-285,.

13. A. Yilmaz, E. Akkaya, E. Damadoglu et al. - "Occult bronchial foreign body aspiration in adults: analysis of four cases," Respirology. 2004; 9(4):561-563.

14. M. Boyd, A. Chatterjee, C. Chiles et al. "Tracheobronchial foreign body aspiration in adults," Southern Medical Journal. 2009; 102(2):171-174. 